\title{
EDITORIAL
}

\section{Responsible conduct of research - Part 1}

Enoka Corea iD https://orcid.org/0000-0003-1450-2098, Himani Molligoda iD https://orcid.org/0000-0002-9216-9035, Co-Editors

Keywords: responsible conduct of research, scientific integrity, research integrity, research misconduct

(C) Authors. This is an open-access article distributed under a Creative Commons Attribution-Share Alike 4.0 International License (CC BY-SA 4.0), which permits unrestricted use, distribution, and reproduction in any medium, provided the original author and source are attributed and materials are shared under the same license.

\section{Introduction}

For many young researchers, mastering research methodology is a challenge. Dealing with the ethical aspects of research poses an additional daunting problem. Navigating the narrow path of responsible conduct of research including adhering to numerous research ethics guidelines, codes of conduct and professional ethical principles can be bewildering.

However, the responsible conduct of research can be simplified into three major areas.

1. Ensuring the wellbeing of the research participant (human or animal)

2. Upholding research integrity

3. Assuring objectivity

The first aspect or 'ethical' research has been dealt with exhaustively. Since research on a 'representative' sample of the population (albeit to generate generalizable knowledge beneficial to all) has the potential for exploitation of research subjects, clear ethics principles and procedures to protect the rights and welfare of research participants have been laid down. Research ethics guidelines, ranging from the Nuremberg Code to the Helsinki Declaration, are well known to any scientist. Institutional review boards or ethics review committees review, approve and monitor research studies following standard operating procedures and it is no longer possible to publish research that has not been approved by such a committee.

The second aspect, i.e., upholding research integrity, is harder to regulate. What we mean by research integrity is the integrity of the research process. This can be defined as adherence to honest and verifiable methods in proposing, performing, evaluating and reporting research activities [1]. While we may think that this is obligatory if scientists are to generate accurate results, breaches of research integrity are increasingly reported, globally. 
The most egregious breaches of research integrity amount to scientific misconduct and may result in retraction of papers, withdrawal of research grants, loss of reputation and even the end of one's career. The National Academy of Sciences of the US limits scientific misconduct to fabrication, falsification, and plagiarism in proposing, performing, or reporting research. Fabrication means making up data or results, falsification is changing data or results and plagiarism is using the ideas or words of another person without giving appropriate credit [1]. Such acts may result in serious punitive action. Genuine errors or differences of opinion in interpretation of data are not considered misconduct [1].

There are other actions that are considered "questionable" research practices not amounting to serious research misconduct. These behaviors are not acceptable and may affect the reputation of the researcher among fellow scientists and the public. Such practices include not maintaining proper research records, failure to retain data for a reasonable period of time, using inappropriate statistical methods in order to enhance the significance of research findings, giving or receiving authorship that is underserved, withholding authorship from individuals who have contributed significantly to the research, refusal to share research materials or data with other researchers etc. In addition, laboratory heads and supervisors may be disciplined for inadequate supervision or exploitation of research students and staff.

We are all aware of scientists who mislead the public by misrepresenting speculations as facts or by releasing preliminary research results to the media without peer review or verification.

There are other examples of conduct that is inappropriate in scientists working in research laboratories. For example, sexual or other types of harassment of staff is viewed very seriously. Misuse or misappropriation of research grants or theft or vandalization of equipment or reagents may lead to indictment and criminal charges. Laboratory staff or the public may be endangered by negligence on the part of a researcher including contravention of government regulations.

Covering up of others' misconduct or reprisals against whistle-blowers who try to alert the institution to such misconduct are also considered offences as well as making false allegations of misconduct in colleagues.

Misconduct in science is wrong, wastes time and resources, can lead to injuries and harmful consequences and undermines public support of science. Misconduct by scientists and weak institutional responses to these incidents can lead to counter-productive regulation and control. Therefore, all researchers should be made aware of responsible conduct of research as part of their basic training in research [2]. The next Editorial will deal with how to achieve responsible conduct of research in actual practice. 
Journal of the Postgraduate Institute of Medicine 2021; 8(1): E143 1-2

http://doi.org/10.4038/jpgim.8345

\section{References}

1. National Academy of Sciences, National Academy of Engineering, and Institute of Medicine. 1992. Responsible Science: Ensuring the Integrity of the Research Process:

Volume I. Washington, DC: The National Academies Press. https://doi.org/10.17226/1864

2. Code of Conduct for Health Research in Sri Lanka (Ministry of Health) (2018) 